Preliminary work

\title{
Study of formulation variables influencing polymeric microparticles by experimental design
}

\author{
Rameshwar K. Deshmukh and Jitendra B. Naik* \\ Department of Pharmaceutical Technology, University Institute of Chemical Technology, North Maharashtra \\ University, Jalgaon, 425 001, Maharashtra, India \\ *Corresponding Author: E-mail: jitunaik@gmail.com; Tel.: +91257 2258441; fax: +91257 2258403
}

Received: December 21,2013; Revised: March 01, 2014; Published: April 01, 2014

\begin{abstract}
The objective of this study was to prepare diclofenac sodium loaded microparticles using the single emulsion [oil-in-water $(o / w)$ ] solvent evaporation method. The $2^{2}$ experimental design methodology was using the Design-Expert software used to evaluate the effect of two formulation variables on the properties of the microspheres in terms of particle size, morphology, encapsulation efficiency, and in vitro drug release. The graphical and mathematical analysis of the design showed that the independent variables had a significant effect on the encapsulation efficiency and drug release of microparticles. The low magnitudes of error and significant values of $R^{2}$ prove the high prognostic ability of the design. The microspheres showed high encapsulation efficiency with an increase in the amount of polymer and a decrease in the amount of PVA in the formulation. The particles were found to be spherical with a smooth surface. Prolonged drug release and enhancement of encapsulation efficiency of polymeric microparticles can be successfully obtained by applying experimental design techniques.
\end{abstract}

Keywords: Microencapsulation; Microparticles; Statistical experimental design; Solvent evaporation; Single emulsion; Drug delivery

\section{Introduction}

Biodegradable and biocompatible microparticles are popularly investigated drug delivery systems for therapeutic drugs [1-5]. They are capable of providing controlled release of the encapsulated drug over a long period of time [6,7]. Encapsulation of the drug within biomaterial-based microparticles can increase the stability of the drug and facilitate controlled and sustained release of the compound [8]. Recently, various different encapsulation techniques have been employed to produce fine particles of various active compounds. Many microencapsulation processes are modifications of the three basic techniques: solvent extraction/evaporation, phase separation (coacervation), and spray-drying techniques [9]. Solvent evaporation and organic phase separation techniques are widely used in the pharmaceutical industry for the preparation of microparticles [1,2,9-12]. These microparticles can reduce side effects, enhance the efficacy of therapy, and increase patient compliance.

Non-steroidal anti-inflammatory drugs (NSAIDs) are usually indicated for the treatment of musculoskeletal disorders where pain and inflammation are present. Diclofenac sodium [sodium(o-((2,6- 
dichlorophenyl)-amino)-phenyl)-acetate] is a NSAID that is commonly used drug for the relief of pain and inflammation in arthritis, rheumatoid arthritis, osteoarthritis, and pain management in case of kidney stones. The dose is 100 to $150 \mathrm{mg}$ daily with a short duration of action. Therefore, repeated dosing of the drug is essential for effective pain management. A major disadvantage of NSAID therapy is the potential for upper gastrointestinal (GI) complications and renal complications [13-19]. Due to the high usage of NSAIDs, a drug delivery formulation which will achieve the highest healing effect with minimal $\mathrm{Gl}$ events is desirable. For that purpose, a sustained release formulation is essential. Therefore, polymeric microparticles of diclofenac sodium might be beneficial to overcome the side effects and increase efficacy of therapy and patient compliance. Diclofenac sodium and ethylcellulose were used as the model drug and polymer for the preparation of microparticles. Ethyl cellulose, an ethyl ether of cellulose, is a long chain polymer of $\beta$-anhydroglucose units joined together by acetal linkages. Ethylcellulose is a hydrophobic polymer widely used in the pharmaceutical field as a coating material for the preparation of microcapsules, microparticles, tablets, and for controlled release dosage forms due to its wide range of practically relevant properties, including low toxicity (FDA approved for internal use), good film forming abilities, and relatively low cost. High viscosity grades are used in drug microencapsulation and for sustained release products [13, 20-23].

Pharmaceutical scientists often using the single factor optimisation technique (one factor at a time) to optimise a formulation. Multiple factor optimisation is more efficient and generates a maximum point (an optimised condition) compared with the single factor optimisation technique. In many process development and manufacturing applications of pharmaceutical formulations, the number of potential input variables (factors) is greater. These formulations often face the challenge of identifying the key input variables or process conditions that affect product quality. However, this challenge can be overcome by using statistical design of experiment tools to carry out the minimum number of experiments to estimate the influence of individual variables on response variables [24-34].

The aim of this study was to evaluate, by means of a statistical experimental design methodology, the influence of the formulation variables on the encapsulation efficiency of diclofenac sodium loaded microparticles in order to obtain a sustained release system to treat musculoskeletal disorders.

\section{Experimental}

\section{Materials}

Diclofenac sodium was provided by Natco Pharma Limited, (Hyderabad, India) as a gift sample. Ethylcellulose was kindly donated by Colorcon India Pvt. Ltd. (Mumbai, India). Polyvinyl alcohol (PVA), ( $M_{w}$ 140,000) was purchased from HiMedia Laboratories Pvt. Ltd. (Mumbai, India). Dichloromethane was obtained from Merck Specialities Pvt. Ltd. (Mumbai, India). Methanol (HPLC Grade) was purchased from RFCL Limited (New Delhi, India). All other solvents and reagents were of analytical grade and used as provided.

\section{Experimental design}

Before application of the design, a number of preliminary trials were conducted to determine the conditions at which the process resulted in microparticles. The levels of factors were also determined by this procedure. The $2^{2}$ (two factors and two levels) factorial design was employed for the optimisation of diclofenac sodium loaded microparticles using the Design-Expert ${ }^{\circledR}$ Software (Version-8.0.7.1, Stat-Ease Inc., Minneapolis, MN) which allows evaluation from four experiments. The purpose of the design was to 
evaluate the effects of the formulation factors and identify the key one that influences the encapsulation efficiency (Dependent variable). The amount of ethylcellulose ( $A, m g$ ), and the amount of PVA (B, \% w/v) were selected as independent variables. The levels of screening variables evaluated in this study are listed in Table 1 along with their low and high levels. Statistical analysis was performed and the significance of the model was determined by the comparisons of statistical parameters [35-37].

Table 1. Screening variables and their levels (coded and actual) used for $2^{2}$ experimental design

\begin{tabular}{lll}
\hline Independent variables & \multicolumn{2}{l}{ Level used, actual (coded) } \\
\cline { 2 - 3 } & Low, $(-1)$ & High, $(+1)$ \\
\hline $\mathrm{A}=$ Amount of ethylcellulose $(\mathrm{mg})$ & 250 & 750 \\
$\mathrm{~B}=$ Amount of PVA as a surfactant $(\% \mathrm{w} / \mathrm{v})$ & 0.05 & 0.2 \\
\hline
\end{tabular}

\section{Preparation of encapsulated microparticles}

Microparticles containing diclofenac sodium were prepared using the oil-in-water $(O / W)$ single emulsion solvent evaporation method. Briefly, $250 \mathrm{mg}$ of diclofenac sodium was mixed with a mixture of dichloromethane $(10 \mathrm{ml})$, and methanol $(10 \mathrm{ml})$ containing a certain proportion of ethylcellulose polymer as per the factorial design. This organic phase was dispersed in $100 \mathrm{ml}$ of distilled water containing the varying amount of PVA as an emulsifier to produce an O/W single emulsion at a speed of 800 RPM using four bladed lab stirrers (Remi Electrotechnik Limited, Thane, India). The external aqueous phase was adjusted to pH 3.9 with acetic acid. Stirring was continued for 3 hours until the evaporation of organic solvent leaving solid microparticles. The microparticles were collected by filtration, rinsed with $\mathrm{n}$-hexane, air dried for 48 hours, and used for further studies.

\section{Characterisation of the microparticles}

The amount of diclofenac sodium encapsulated into microparticles was determined by UV-Vis spectrophotometer (HITACHI U-2900, Tokyo, Japan). An accurately weighed $10 \mathrm{mg}$ of microparticles were stirred with dichloromethane $(2 \mathrm{ml})$ to dissolve the polymeric coat, and was then extracted in phosphate buffer solution (pH 6.8). Stirring continued for $30 \mathrm{~min}$ at room temperature to facilitate the evaporation of organic solvent. The dispersion was filtered, and the residue was washed with phosphate buffer solution. The encapsulation efficiency was determined in the filtrate after appropriate dilution with a phosphate buffer solution at $276 \mathrm{~nm}$ using a UV-Vis spectrophotometer. The encapsulation efficiency was expressed as the percentage of drug incorporated in the formulation relative to the total amount of drug used in the formulation. The encapsulation efficiency of diclofenac sodium was calculated using the following equation:

\section{$\%$ Encapsulation efficiency $=\frac{[\text { weight of drug determined }]}{[\text { weight of drug added }]} \times 100$}

Of all the formulations, microparticles with a higher encapsulation efficiency were chosen for further characterisation study.

The particle size and shape of the drug loaded microspheres was determined by capturing the series of digital images using Motic B1 advanced series digital microscope (Motic, China) fitted with imaging accessories equipped with computer-controlled image analysis software (Motic Images Plus, $2.0 \mathrm{ML}$ Version). Calibration of an object lens was done using a standard calibrated circle. The sample was put on the slide, and the diameter of the particles was determined. 
Drug release from microparticles was performed in vitro using phosphate buffer $(\mathrm{pH} \mathrm{6.8)} \mathrm{for} 12$ hours in a tablet dissolution tester (Electrolab TDT-06 T, India) USP XXVIII, type I (100 RPM, $37 \pm 0.5^{\circ} \mathrm{C}$ ). The dissolution medium of phosphate buffer $(\mathrm{pH}$ 6.8) was prepared according to the Indian Pharmacopoeia [38]. Aliquots of the dissolution medium were withdrawn at predetermined intervals and replenished with fresh dissolution media to maintain the sink condition. The samples were filtered through a Whatman filter paper no. 41. The diclofenac sodium content of each sample after suitable dilution was assayed by UV-Vis spectroscopy at $\lambda_{\max }$ of $276 \mathrm{~nm}$ using a $1 \mathrm{~cm}$ cell. The experiments were done in triplicate. The release data were evaluated kinetically to study the possible mechanism of drug release from the microparticles.

\section{Results and Discussion}

\section{Experimental design}

The statistical experimental design is a useful and efficient mathematical approach to evaluate the effect of a factor on a particular response generated by conducting a smaller number of experimental trials. Each analysed variable was evaluated at two levels, high $(+1)$ and low $(-1)$. The evaluation consisted of analysing the response in all the conditions quoted in Table 2. The polynomial model describing the correlation between the formulation process variables and encapsulation efficiency can be represented by the following equation:

$$
\text { E.E. }=72.68+3.83 \mathrm{~A}-8.68 \mathrm{~B}
$$

In the above equation a negative sign of variable $B$ signifies the antagonistic effect, while a positive sign of variable A signifies a synergistic effect to the responses. It can be observed from the above equations that \% E.E. was influenced by both factors. From the ANOVA of design for encapsulation efficiency, $p$-value, F-value, mean square and $R^{2}$ of the model are given in Table 3.

Table 2. The $2^{2}$ experimental design matrix (in coded level) and experimental results

\begin{tabular}{cccc}
\hline Run & \multicolumn{2}{c}{ Independent variables } & Encapsulation Efficiency \\
\cline { 2 - 3 } & A & B & \\
\hline 1 & 750 & 0.05 & $84.93 \pm 0.06$ \\
2 & 750 & 0.2 & $68.08 \pm 0.04$ \\
3 & 250 & 0.2 & $59.91 \pm 0.12$ \\
4 & 250 & 0.05 & $77.78 \pm 0.01$ \\
\hline
\end{tabular}

Table 3. Analysis of variance for encapsulation efficiency

\begin{tabular}{lccccc}
\hline Source & $\begin{array}{c}\text { Sum of } \\
\text { squares }\end{array}$ & d.f. & Mean square & F value & $\begin{array}{c}p \text {-Value } \\
\text { Prob }>\mathrm{F}\end{array}$ \\
\hline Model & 359.87 & 2 & 179.94 & 691.51 & $0.0269(\mathrm{~S})$ \\
A- Ethylcellulose & 58.68 & 1 & 58.68 & 225.50 & $0.0423(\mathrm{~S})$ \\
B- PVA & 301.20 & 1 & 301.20 & 1157.52 & $0.0187(\mathrm{~S})$ \\
Residual & 0.26 & 1 & 0.26 & & \\
Total (Corrected) & 360.13 & 3 & & & \\
Correlation coefficient $\left(R^{2}\right)$ & 0.9993 & & & & \\
Adjusted $R^{2}$ & 0.9978 & & & & \\
Predicted $R^{2}$ & 0.9884 & & & & \\
PRESS & 4.16 & & & & \\
d.f.
\end{tabular}

d.f. degree of freedom, ${ }^{\mathrm{S}}$ Significant 
Standardised Pareto charts, (Fig. 1) representing the estimated effects of parameters on response, can allow us to check the statistical significance of experimental design. It consists of bars with a length proportional to the absolute value of the estimated effects divided by the standard error, which is the $t$ value of the student's t-test. It can be observed that ethylcellulose and PVA have significant influence on the encapsulation efficiency.

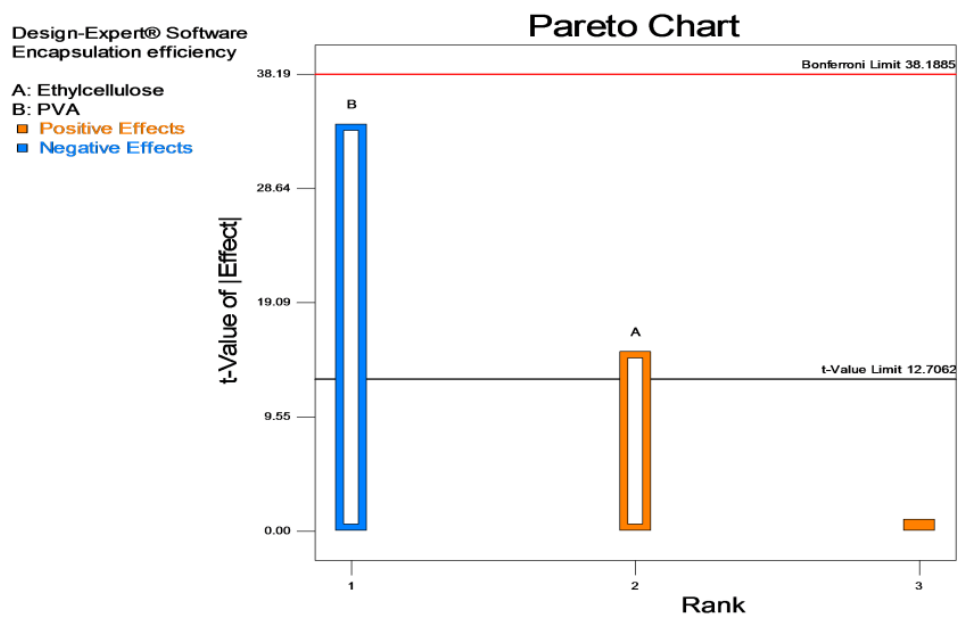

Figure 1. Pareto chart of standardised effects on the encapsulation efficiency

\section{Characterisation of the microparticles}

The encapsulation efficiency of different experimental runs of microparticles is reported in Table 2. Two-dimensional (2D) contour plots and three-dimensional (3D) response plots resulting from the equations were constructed to visualise the effect of independent variables on the response using DesignExpert software [39]. It was observed that the encapsulation efficiency was higher with a high level of ethylcellulose and low level of PVA. Surface response plots and contour plots (Fig. 2 A \& B) represent the increase in \% encapsulation efficiency with an increase in the amount of ethylcellulose and a decrease in the amount of PVA.
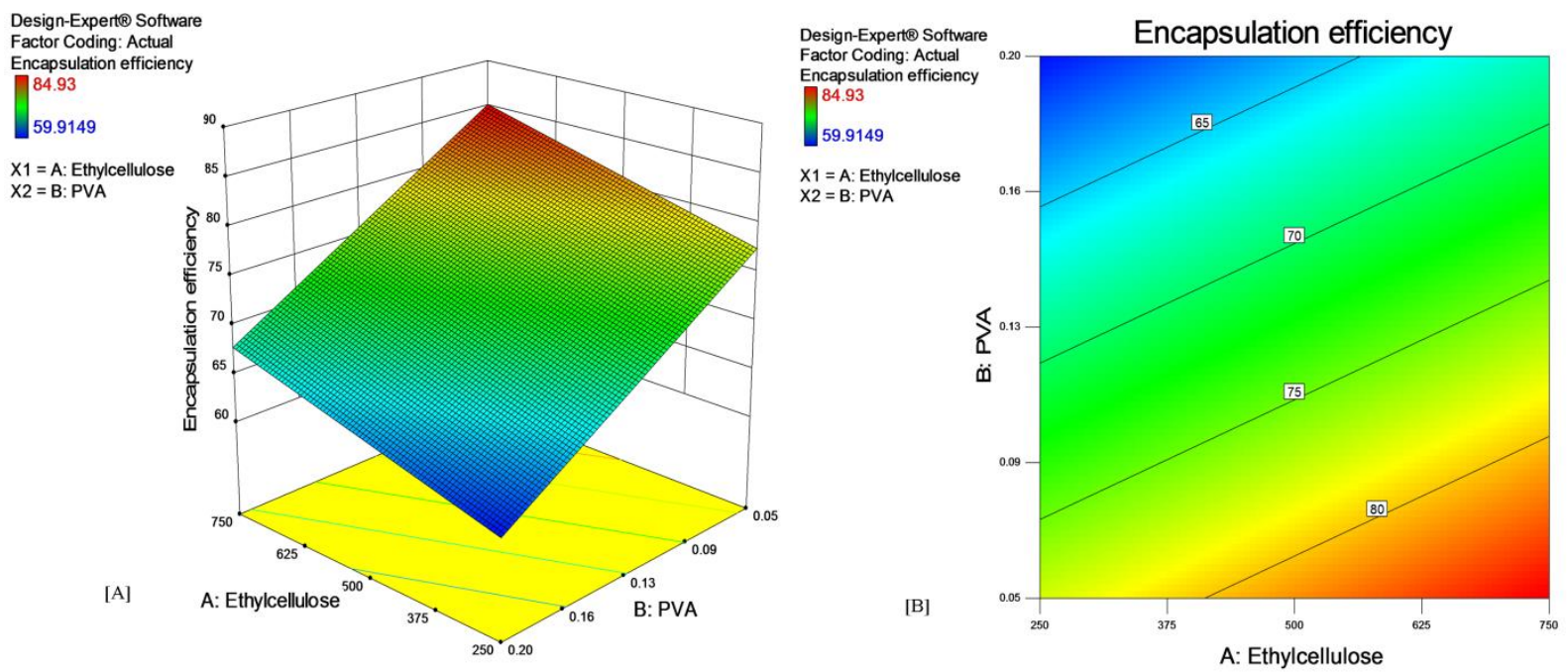

Figure 2. Response surface plot $[A]$ and contour plot $[B$,$] showing the effect of independent variables on response$

The shape, size, and surface morphology of the diclofenac sodium loaded microparticles were examined by Motic microscope. In this study, the particle size of the drug loaded microspheres captured 
using Motic ${ }^{B 1}$ advanced series digital microscope (Fig. 3) was found to be in the range of $10-200 \mu \mathrm{m}$. The particles were found to be discrete with spherical geometry.

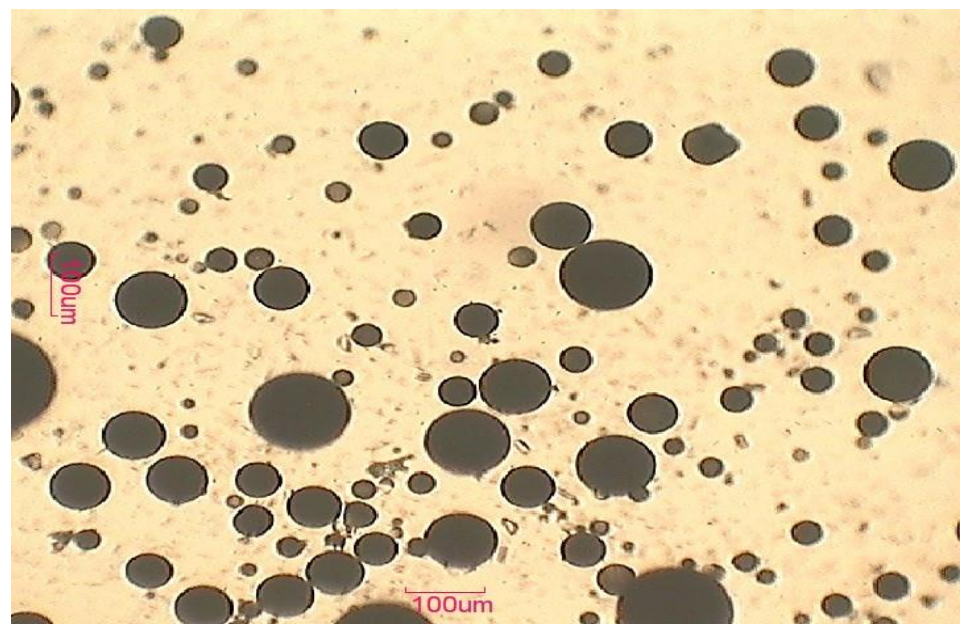

Figure 3. Image of microparticles by Motic microscope

Ethylcellulose is a water insoluble polymer able to control drug release from microparticles. Fig. 4 shows the cumulative \% drug release as a function of the dissolution time from the diclofenac sodium loaded microparticles. The release of diclofenac sodium was evaluated using phosphate buffer $(\mathrm{pH} \mathrm{6.8)}$ as the release media. Prolonged drug release (92 \%) (Fig. 4) from the microparticles was observed over 12 hours. The dissolution data were fitted into the Matrix model equation. The sample showed good linearity $\left(R^{2}: 0.9764\right)$ with a value of the slope $(n)<0.45$. It indicates that Fickian diffusion is the dominant mechanism of drug release from these microspheres.

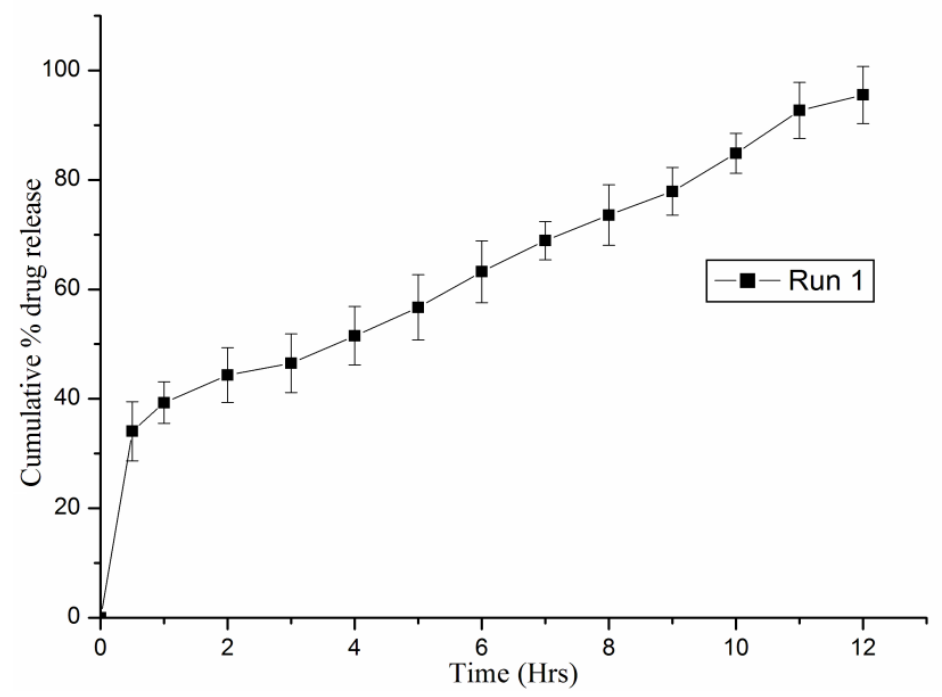

Figure 4. Cumulative \% drug release from the microspheres

\section{Conclusions}

This work shows that diclofenac sodium loaded ethylcellulose polymeric microparticles were successfully prepared. The effects of formulation variables had been screened out by employing $2^{2}$ experimental design. The formulation variables have a significant effect on the encapsulation efficiency of microspheres. Design and optimisation through statistical experimental designs work well in the 
development of polymeric microparticles. Microparticles prepared by such methods may represent a promising approach for the enhancement of encapsulation efficiency of diclofenac sodium.

Acknowledgements: Authors are very much thankful to University Grants Commission, New Delhi for providing financial assistance to carry out this research work. The authors gratefully acknowledge Natco Pharma Limited, (Hyderabad, India), Evonik Degussa India Pvt. Ltd. (Mumbai, India) \& Colorcon India Pvt. Ltd. (Mumbai, India) for providing the gift sample of diclofenac sodium \& ethylcellulose respectively.

\section{References}

[1] A. Lamprecht, H.R. Torres, U. Schafer, C.M. Lehr, Journal of Controlled Release 69 (2000) 445-454.

[2] J. Herrmann, T. Bodmeier, International Journal of Pharmaceutics 126 (1995) 129-138.

[3] H.S. Yoo, Colloids and surfaces B: Biointerfaces 52 (2006) 47-51.

[4] E. Polard, P.S. Corre, F. Chevanne, R.L. Verge, International Journal of Pharmaceutics 134 (1996) 3746.

[5] J.B. Naik, R.K. Deshmukh, V.V. Kamble, American Journal of PharmTech Research 3 (2013) 892-904.

[6] K. Avneshi, S.K. Yadav, S.C. Yadav, Colloids and Surfaces B: Biointerfaces 75 (2010) 1-18.

[7] S. Raveendran, A.C. Poulose, Y. Yoshida, T. Maekawa, D.S. Kumar, Carbohydrate Polymer 91 (2013) 22-32.

[8] A. Kochut, P. Dersch, European Journal of Pharmaceutics and Biopharmaceutics 84 (2013) 242-250.

[9] S. Freitas, H.P. Merkle, B. Gander, Journal of Controlled Release 102 (2005) 313-332.

[10] R. Bodmeier, H. Chen, P. Tyle, P. Jarosz, Journal of Controlled Release 15 (1991) 65-77.

[11] M. Li, O. Rouaud, D. Poncelet, International Journal of Pharmaceutics 363 (2008) 26-39.

[12] H. Heiskanen, P. Denifl, P. Pitkanen, M. Hurme. Chemical Engineering Research and Design 90 (2012) 1517-1526.

[13] J.L. Arias, M. Lopez-Viota, J. Lopez-Viota, A.V. Delgado, International Journal of Pharmeutics 382 (2009) 270-276.

[14] J.T. Edelson, A.N. Tosteson, P. Sax, JAMA 264 (1990) 41-47.

[15] C.H. Barrier, B.I. Hirschowitz, Arthritis Rheum 32 (1989) 926-932.

[16] T. Pincus, M. Griffin, American Journal of Medicine 91 (1991) 209-212.

[17] C. Sostres, C.J. Gargallo, M.T. Arroyo, A. Ganas, Best Practice in Research and Clinical Gastrointerology 24 (2010) 121-132.

[18] G. Thiefin, L. Beaugerie, Joint Bone Spine 72 (2005) 286-294.

[19] A. Pilotto, D. Sancarlo, F. Addante, C. Scarcelli, M. Franceschi, Surgical Oncology 19 (2010) 167-172.

[20] R.C. Rowe, P.J. Sheskey, M.E. Quinn. Handbook of pharmaceutical excipients. 6 ed. Washington, DC, USA: Pharmaceutical Press and American Pharmacists Association; 2009.

[21] H.L. Lai, K. Pitt, D.Q.M. Craig, International Journal of Pharmaceutics 386 (2010) 178-184.

[22] M.A. Alhnan, A.W. Basit, European Journal of Pharmaceutical Sciences 42 (2011) 30-36.

[23] M. Song, N. Li, S. Sun, L.R. Tiedt, W. Liebenberg, M.M. deVilliers, II Farmaco 60 (2005) 261-267.

[24] J. Malakar, A.K. Nayak, Chemical Engineering Research and Design 90 (2012) 1834-1846.

[25] D. Bas, I.H. Boyaci, Journal of Food Engineering 78 (2007) 836-845.

[26] S. Chopra, G.V. Patil, S.K. Motwani. European Journal of Pharmaceutics and Biopharmaeutics 66 (2007) 73-82.

[27] S. Kehoe, N. Kilcup, D. Boyd, Material Letters 86 (2012) 13-17.

[28] R.S. Liu, Y.J. Tang, Bioresource Technology 101 (2010) 3139-3146. 
[29] X. Li, J. Ouyang, Y. Xu, M. Chen, X.Y. Song, Q. Yong. Bioresource Technology 100 (2009) 3139-3146.

[30] N. Kettaneh-Wold, Journal of Pharmaceutical and Biomedical Analysis 9 (1991) 605-610.

[31] R. Ragonese, M. Macka, J. Hughes, P. Petocz, Journal of Pharmaceutical and Biomedical Analysis 27 (2002) 995-1007.

[32] S.A. Martins, D.A. Prazeres, L.P. Fonseca, G.A. Monteiro, Analytical Biochemistry 391 (2009) 17-23.

[33] S. Jose, J.F. Fangueiro, J. Smitha, T.A. Cinu, A.J. Chacko, K. Premaletha, E.B. Souto, Colloids and Surfaces B:Biointerfaces 92 (2012) 175-179.

[34] R.K. Deshmukh, J.B. Naik, Journal of Pharmaceutical Innovation 8 (2013) 276-287.

[35] L. Al Haushey, M.A. Bolzinger, C. Bordes, J.Y. Gauvrit, S. Briancon, International Journal of Pharmaceutics 344 (2007) 16-25.

[36] S. Bolton, C. Bon, Pharmaceutical Statitics Practical and Clinical Application. 5 ed. New York, London: Informa Healthcare; 2009.

[37] S.A. Joshi, S.S. Chavhan, K.K. Sawant, European Journal of Pharmceutics and Biopharmaceutics 76 (2010) 189-99.

[38] Indian Pharmacopoeia Commission Ghaziabad, India. Indian Pharmacopoeia. 2010. pp. 562.

[39] A.K. Nayak, D. Pal, J. Pradhan, M.S. Hasnain, International Journal of Biological Macromolecules 54 (2013) 144-54.

(C)2014 by the authors; licensee IAPC, Zagreb, Croatia. This article is an open-access article distributed under the terms and conditions of the Creative Commons Attribution license (http://creativecommons.org/licenses/by/3.0/) (cc) Br 